\title{
BIBECHANA
}

A Multidisciplinary Journal of Science, Technology and Mathematics ISSN 2091-0762 (Print), 2382-5340 (Online)

Journal homepage: http://nepjol.info/index.php/BIBECHANA

Publisher: Research Council of Science and Technology, Biratnagar, Nepal

\section{Study of electronic transport parameters on the heterojunction of AlGaN/GaN, grown on Sapphire: two layer model}

\author{
S. Shrestha ${ }^{1 *}$, Ambika Shakya1, C. K. Sarkar ${ }^{2}$ \\ ${ }^{1}$ Central Department of Physics, Tribhuvan University, Kathmandu, Nepal \\ ${ }^{2}$ Dept. of ETCE, Jadavpur University, Kolkata-700032, India \\ *Email: sanju12np@yahoo.com \\ Article history: Received 17 August, 2018; Accepted 11 September, 2018 \\ DOI: http://dx.doi.org/10.3126/bibechana.v16i0.21072 \\ This work is licensed under the Creative Commons CC BY-NC License. \\ https://creativecommons.org/licenses/by-nc/4.0/
}

Abstract

In spatially confined system such as heterojunction of high and low band gap material, carriers are transferred from higher band gap to the lower band gap. It causes the band bending and the formation of a triangular quantum well at the junction. Such system behaves as quantum-two dimension (Q2D) system because the carriers are free to move on a plane, perpendicular to the junction. Mobility of such quantized system is very high as compare to the bulk system due to the reduction of various scattering mechanisms. GaN is a very useful material. However, a non availability of single crystalline form of GaN and perfectly matched substrates are always problems for GaN. Hence GaN, grown on a substrate such as sapphire is having a very large dislocations at the interface. Such interfacial layer significantly affects the transport parameters of the material, where the transport properties are highly dominated by scattering due to dislocations. The authors have calculated the mobilities of $\mathrm{AlGaN} / \mathrm{GaN}$, a heterojunction considering the GaN, grown on Sapphire with reference to the two layer model of Look, in which the 2 nd layer is the dislocation layer of $\mathrm{GaN}$ and the $1^{\text {st }}$ layer is the junction of $\mathrm{AlGaN} / \mathrm{GaN}$ where carriers are in the form of two dimensional electron gas (2D EG). The obtained calculated results are also compared with the experimental results as obtained by Sibel Gokden et al. It is observed that the nature of the curve is found to be in agreement with the experimental curve when the ratio of the thicknesses is taken to be $1: 1$.

Keywords: Heterojunction; Quantum well; Two layer model; AlGaN/GaN.

\section{Introduction}

In the present and in the upcoming generation, there is very high demand of high speed electronic devices. Hence, the study of electron transport in low dimensional semiconductor system has been very important. The low dimensional devices can be made either by external perturbation or by spatial confinement. The development of various growth techniques such as molecular beam epitaxy (MBE) 
and molecular oxide vapor chemical deposition (MOVCD) has opened the door for the spatially confined structures like quantum wells (QWs), quantum well wires (QWWs) and quantum dots (QDs).

Heterojunction: The idea of heterojunction was forwarded by Anderson[1]. Heterojunction is the junction between the low and high band gap materials as shown in Fig. 1 (a), in which the electrons from higher band gap fall to the lower band gap. The accumulated electrons in the lower band gap create space charges in the higher band gap region and the band bending takes place due to Coulomb interaction between positive charges left on donor side and the electrons in the interface. As a result, a triangular well is formed at the junction. Here, the electrons get trapped into the well and quantized in the various energy levels shown by Fig. 1(b). Such quantized electrons can move only in the plane of the quantized level or perpendicular to the surface of the junction. These quantized electrons are called two dimensional electron gas (2D EG).

In the present work the authors have used the heterojunction of $\mathrm{AlGaN} / \mathrm{GaN}$ in which from doped and has higher band gap AlGaN electron get transferred to the undoped and lower band gap GaN. These electrons are quantized in various levels forming 2D EG as described above. However, the authors have taken account of the extreme quantum limit (EQL) condition i.e. only the electrons in the lowest energy level take part in the various electronic transport phenomena.

In compound semiconductors, the scattering of the electrons by ionized impurities limits the mobility of the sample [2]. Hence, the ionized impurity (ii) is also included for the heterojunction. At the same time due to the presence of interface between $\mathrm{GaN}$ and $\mathrm{AlGaN}$ surface roughness scattering mechanism (sr) plays an important and dominating role in limiting the mobility at low temperatures and high carrier densities. The acoustic phonon (ac) via deformation potential scattering mechanism is caused by the perturbing potential arising from the strain produced in the crystal lattice by the acoustic vibrations. In crystals lacking inversion symmetry, other than the deformation potential interactions, piezoelectric coupling occurs with acoustic modes. The displacements of the ionized atoms associated with the acoustic vibrations in these crystals produce an electrical polarization giving rise to piezoelectric scattering (pz) of the carries. Piezoelectric scattering (pz) is important for weak-field transport at low temperatures[3]. At high fields however, the important of this type of scattering deceases since the corresponding scattering rate diminishes with increasing electron energy. Hence, various low temperature scatterings mechanisms such as acoustic phonon (ac), piezoelectric (pz), surface roughness (sr) and ionize impurity (ii) scattering etc are included to the heterojunction of $\mathrm{AlGaN} / \mathrm{GaN}$.

Two Layer Model: GaN is not available in single crystalline form. Therefore, it is normally grown on sapphire $\left(\mathrm{Al}_{2} \mathrm{O}_{3}\right)$ or $\mathrm{SiC}$ substrate with a large lattice mismatch. The $\mathrm{GaN}$ grown on substrate sapphire having $14 \%$ lattice size mismatch and 34\% mismatch in the thermal expansion coefficient ${ }^{4}$ has been selected for the present work.

Hence, the whole material $\mathrm{AlGaN} / \mathrm{GaN}$ grown on sapphire substrate system can be considered to be made up of two layers ${ }^{5}$ formulated by Look et al. The first layer is the junction between AlGaN/GaN and the second layer is the dislocation layer between $\mathrm{GaN}$ and the sapphire substrate as shown in the Fig.2. According to the model, current passing through the whole system can be divided into two layers as if two resistors are kept in parallel. The combined current will be the sum of the currents due to two individual layers, as shown in the Fig. 2. The model has been used to calculate different transport parameters such as ac and dc mobilities and conductivities. 


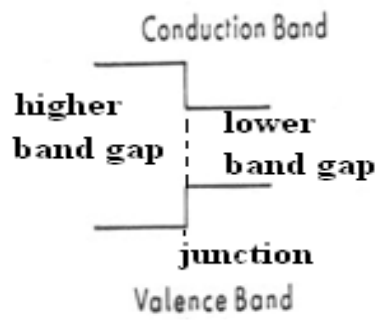

Fig. 1 (a) Heterojuction of higher and lower band gap semiconductor.

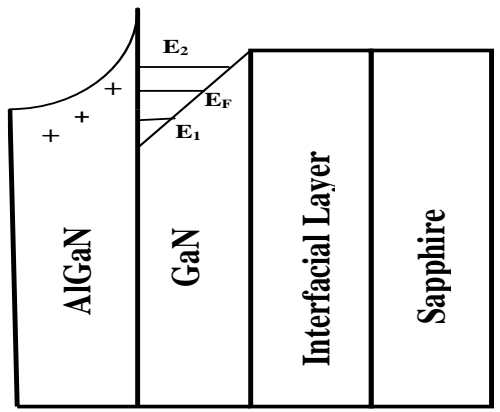

Fig. 2(a)

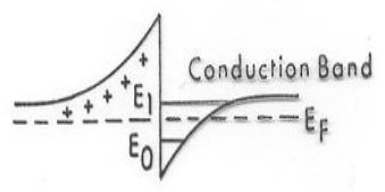

Fig. 1 (b): Quantized energy levels at heterojunction.
Fig. 2(a)

Fig. 2 (a) and Fig 2 (b) represent the schematic representation of the two layer model of the $\mathrm{AlGaN} / \mathrm{GaN}$ grown on sapphire. The top or the first layer represents the junction of $\mathrm{AlGaN} / \mathrm{GaN}$ heterojunction while the second layer represents the interfacial layer of $\mathrm{GaN}$ and the sapphire. The bottom most layer is the substrate of sapphire which is an insulator layer and does not take part in any electronic transport phenomena.

Due to the high density of dislocations in the interfacial layer, transport properties in the layer are highly affected by scattering due to the dislocation. Hence scattering due to dislocation will be dominant in the interfacial layer whereas various other types of scattering mechanisms are included for the heterojunction such as ionized impurity (ii), piezoelectric (pz) acoustic phonon (ac) and surface roughness (sr) scatterings as mentioned above[6]. The influence of dislocation scattering (dis) in the heterojunction layer has not been considered because of the negligible density of dislocations as compared to that of the interfacial layer.

\section{Theory}

Transport Parameters Based on Two Layer Model: Due to the mismatch between the GaN and the sapphire, very large number of dislocations is present in the interfacial layer. The transport parameters are highly affected by these dense dislocations. However, in the heterojunction, the transport 
parameters are affected by various other phonon and nonphonon scattering mechanisms as described above.

According to the two- layer model, the combined current will be the sum of the currents through two individual layers. Hence it is given as:

$$
I_{\text {com }}=I_{1}+I_{2}
$$

where, $I_{1}, I_{2}$ and $I_{\text {com }}$ are current in the heterojunction, the interfacial layer and the total current respectively. The subscripts 1 and 2 are used for the heterojunction or the first layer and the interfacial layer or the second layers respectively in the rest of the work.

Combined Mobility: We have, current density, $J=I / A$ or $J=\sigma E \mathrm{~J}, A=b \times W, E=V / l$, $\sigma=n e \mu$ and $\mu=\frac{v_{e}}{E}$. Therefore

$$
I=n e \mu V A / l
$$

Now, substituting the values of currents for the heterojunction, the interfacial layer and the combined two layer with their respective parameters from equation in Eq.1, we get the combined mobility $\mu_{c o m}$ as

or

$$
n e \mu_{c o m} \frac{V}{l} b W=n_{1} e \mu_{1} \frac{V}{l} b W_{1}+n_{2} e \mu_{2} \frac{V}{l} b W_{2}
$$

$$
\mu_{\text {com }}=\frac{n_{1} a \mu_{1}+n_{2} \mu_{2}}{n_{1} a+n_{2}}
$$

where, $\mathrm{a}=\left(\mathrm{W}_{1} / \mathrm{W}_{2}\right)$ : the ratio of the thickness of the heterojunction $\mathrm{W}_{1}$ to the interfacial layer $\mathrm{W}_{2}$.

For the heterojunction, as the first layer the mobility is given as:

$$
\mu_{1}=\frac{e}{m^{*}}\langle\tau\rangle
$$

where $\langle\tau\rangle$ is the average of the total relaxation time, it is the sum of individual scattering mechanism due to ionized impurity (ii), piezoelectric (pz) acoustic phonon (ac) and surface roughness (sr) scatterings as mentioned above[6].

For the, dislocation layer i.e. the 2 nd layer $\langle\tau\rangle$ in the Eq. 3 displaced by $\left\langle\tau_{d i s}\right\rangle$ and obtained the mobility for the layer. Where $\tau_{d i s}$ is the relaxation time for dislocation scattering mechanism [7].

In the presence of a weak ac field with frequency, , the scattering time or relaxation time becomes complex, i.e $\omega^{-1}$ changes to $(\tau+i \omega)^{-1}$ leading to the real and the imaginary parts of the mobility $\mu_{r}$ and $\mu_{i m}$ respectively. These are given as[8]:

$$
\begin{aligned}
& \mu=\mu_{r}+\mu_{i m} \\
& \mu_{r}=\left(\frac{e}{m^{*}}\right)\left\langle\frac{\tau}{1+\omega^{2} \tau^{2}}\right\rangle \\
& \mu_{i m}=\left(\frac{e}{m^{*}}\right) \omega\left\langle\frac{\tau}{1+\omega^{2} \tau^{2}}\right\rangle
\end{aligned}
$$

where $\omega$ is the angular frequency and $\tau$ is the scattering mechanisms as described above.

The real and imaginary parts of ac mobility given by Eqs. 4 and 5 have been evaluated for the heterojunction and the interfacial layers separately by assuming their respective dominant scattering mechanisms as explained earlier and, the combined real part $\left(\mu_{r c o m}\right)$ and the combined imaginary part $\left(\mu_{\text {imcom }}\right)$ of the ac mobility are obtained as it has been carried out in the case of dc mobility i.e. by using Eq. 2 with the real part of the respective mobilities and the imaginary part of the respective mobilities respectively. 


\section{Results and Discussion}

The alloy composition $\mathrm{x}$ for $\mathrm{AlxGa}(1-\mathrm{x}) \mathrm{N}$ is taken to be 0.33 . The dislocation density in the interfacial layer (ndis), carrier concentration in the triangular well (n2D) and the interfacial layer (ns) are taken as 7x1014 m-2[4], $1014 \mathrm{~m}-2$ and $1016 \mathrm{~m}-2$ [9] respectively.

Fig. 3 represents the variation of dc mobility with temperature in the heterojunction of $\mathrm{AlGaN} / \mathrm{GaN}$ for various scattering mechanisms such as acoustic phonon via deformation potential (ac), surface roughness (sr), ionized impurity (ii), piezoelectric (pz) scattering [6] and dislocation (dis) scattering mechanisms [7] individually.

In the Fig. 3, it is seen that the mobility increases significantly with rise in temperature for non phonon scattering mechanisms like ionize impurity (ii) and surface roughness scatterings (sr), shown by curves indicated by 'ii' and 'sr' respectively in the Fig. The figure shows that at low temperature below $30 \mathrm{~K}$ the ionize impurity scattering mechanism dominates all other scattering mechanisms. That means the non phonon scattering mechanism is dominant at low temperature as compared to that of the phonon scattering mechanisms. At temperature above $30 \mathrm{~K}$, the piezoelectric scattering mechanism (pz) is dominant. This indicates that the phonon scattering dominates the other non phonon scattering mechanisms at temperature above $30 \mathrm{~K}$. The mobility due to dislocation scattering in the junction between $\mathrm{GaN}$ and sapphire is constant with temperature, shown by the curve indicated as (dis) in the Fig.

Fig. 3 shows the variation of 2D EG dc mobility versus temperature in the heterojunction of $\mathrm{AlGaN} / \mathrm{GaN}$ including the total effect of all scattering mechanisms. The total mobility $\mu_{2 \mathrm{D}} \mathrm{EG}$ in the heterojunction initially rises with temperature, reaches maximum at $41.8 \mathrm{~K}$ and then decreases with the increase of temperature. The maximum value of such mobililty is obtained to be $0.57516 \mathrm{~m} 2 / \mathrm{Vs}$.

The curve $\mu$ dis shows the variation of the interfacial layer mobility with temperature for dislocation layer or the interfacial layer of $\mathrm{GaN}$ and the sapphire substrate. The interfacial layer mobility due to dislocation scattering is constant of temperature. Also, the magnitude of the dislocation scattering mechanism is dominant at all temperature as compared to all other scattering mechanisms in the heterojunction.

The curves indicated as $\mu_{24: 1}$ and $\mu_{1: 1}$ is shown in the Fig.4 are combined mobilities taking the ratios of the thickness of the heterojunction and the dislocation layer to be 24:1 and 1:1 respectively. Both the mobility curves are observed to be increased with temperature, reaches maximum value at about $42 \mathrm{~K}$ and then decreases with the increase of temperature. However, the slope of the mobility $\mu_{1: 1}$ decreases gradually with the increase of temperature in the higher temperature region.

Comparison of theoretical mobility curves such as $\mu_{24: 1,}, \mu_{1: 1}$ and $\mu$ hetero with experimental mobility curve $\mu_{\exp }$ [10] is shown in Fig 5. Considering the heterojunction as a single layer, in which all the scattering mechanism such as ionized impurities (ii), surface roughness (sr), acoustic phonon via potential deformation (ac), piezoelectric (pz) and the dislocation scattering (dis) are obtained individually and the total of which is obtained by using Matthiessen's rule for the heterojunction of AlGaN/GaN. The author Gokden [10] has compared his experimental Hall mobility of a 2D EG formed at $\mathrm{AlGaN} / \mathrm{GaN}$ heterointerface with the theory. In which the author has also considered all standard scattering mechanisms including dislocation scattering (dis), acoustic deformation via potential scattering (ac), piezoelectric (pz), polar optical phonon (pop), background and remote impurities (ii) in the heterojunction of $\mathrm{AlGaN} / \mathrm{GaN}$. 
In the Fig. 6 the magnitude of the curves $\mu_{1: 1}$ and $\mu_{24: 1}$ at low temperature range is comparable with that of the curve $\mu \exp$ through the temperature range. The curves $\mu_{1: 1}$ and $\mu_{24: 1}$ initially increase to certain value then gradually decrease, whereas where as the curve $\mu_{\text {exp }}$ decreases rather steeply with the increase of temperature. The curve $\mu_{24: 1}$ has hump like nature at temperature around $40 \mathrm{~K}$ that doesn't match with $\mu_{\exp }$ exactly. Hence the curve $\mu_{1: 1}$ is much comparable with $\mu_{\exp }$ though at high temperature its magnitude is slightly different. The reason behind the difference of magnitudes at high temperature is expected due to that in the present work, the optical phonon scattering mechanism (pop) is not considered. At high temperature, polar optical scattering (pop) is much effective due to which the mobility decreases with temperature. Hence at present work, the variation of mobility is taken for temperature range limited up to $140 \mathrm{~K}$ only. Further inclusion of polar optical phonon (pop) scattering mechanism is expected to get the theoretical result more closed to the experimental result.

However, the figure shows that the two layer model gives the results closer to the experimental value rather than the single layer concept. Moreover, the experimental mobility curve agrees with the combined mobility for the junction to the dislocation layer thickness ratio 1:1 rather than the ratio 24:1. This signifies that the consideration of dislocation layer as a separate layer which has thickness equal to that of the junction in the two layer model is more effective and the results obtained is convincing with the experimental data also.

The variation of the temperature dependent two layer ac mobility at constant frequency $60 \mathrm{GHz}$ for the junction to interfacial layer thickness in the ratio 1:1 with various scattering mechanisms in the heterojunction as mentioned above and the dislocation scattering only in the interfacial layer is shown in Fig 6. The combined real part of ac mobility indicated by the curve $\mu_{\mathrm{r}}$ first increases to the highest at temperature $42 \mathrm{~K}$ and then decreases gradually with the increase of the temperature. The highest value of the real part is nearly equal to that of the combined dc mobility for the ratio 1:1 as shown in the Fig 5. In Eq. (4), when $\omega$ is taken as zero, the real part of the ac mobility determines the dc mobility term which is true in low frequency region. Similarly the combined imaginary part of the ac mobility indicated by the curve $\mu_{\mathrm{im}}$ in the Fig 6 first increases at about temperature $42 \mathrm{~K}$ then it decreases gradually as temperature increases.

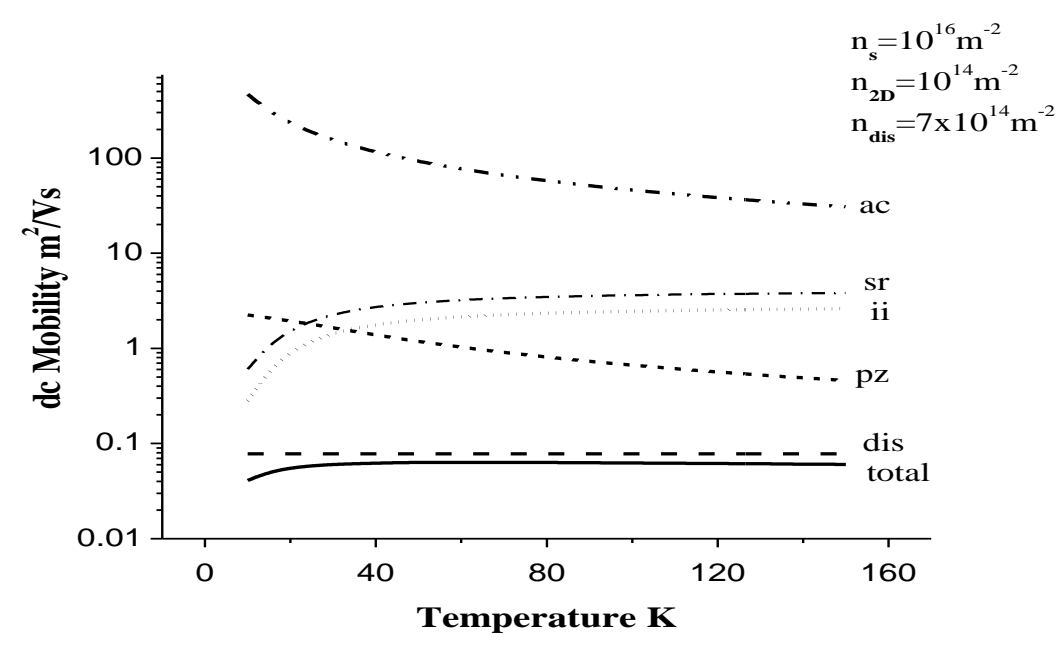

Fig. 3: Variation of dc mobility with temperature in the heterojunction of $\mathrm{AlGaN} / \mathrm{GaN}$ for various scattering mechanisms such as acoustic phonon (ac),piezoelectric (pz), surface roughness (sr), ionized impurity (ii): and only the dislocation (dis) scatterings for interfacial layer separately and also the resultant of above all scattering mechanisms(total) in the heterojunction layer considering it as a single layer. 


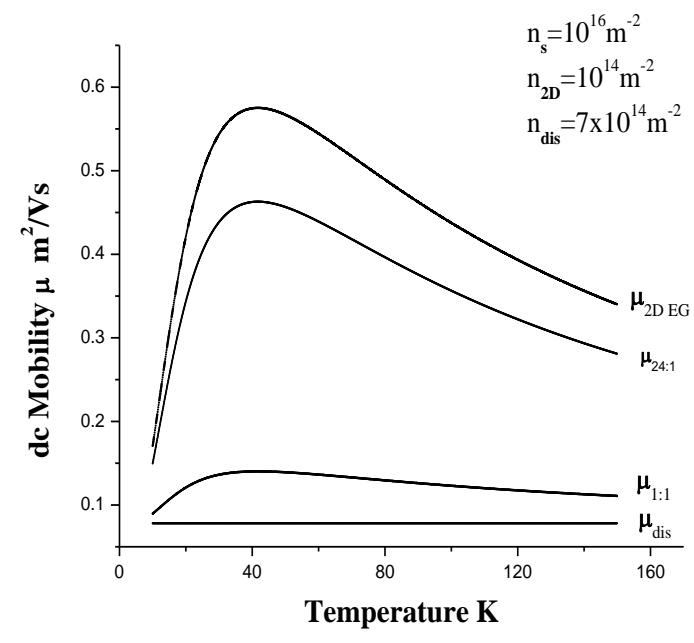

Fig. 4: Variation of 2D EG mobility $\mu_{2 D E G}$ versus temperature in which curves shown by $\mu_{24: 1}, \mu_{1: 1}$ $\mu_{2 \mathrm{D} \mathrm{EG}} \mu_{\mathrm{dis}}$ are for the resultant taking the ration of thickness of heterojunction to the interfacial layer 24:, 1:1, the heterojunction mobility alone and the interfacial mobility alone respectively.

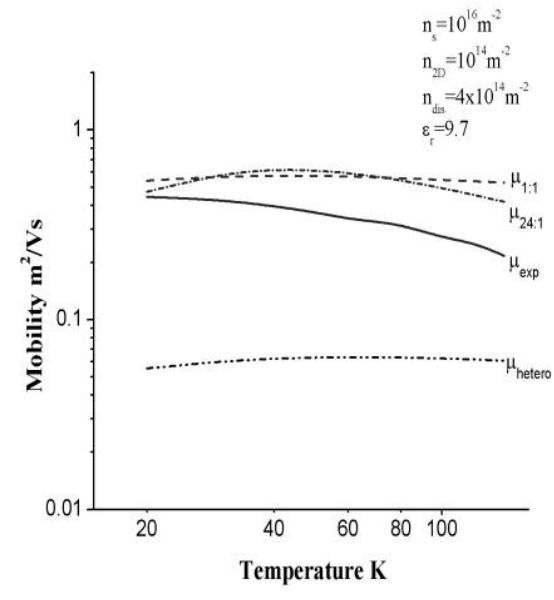

Fig. 5

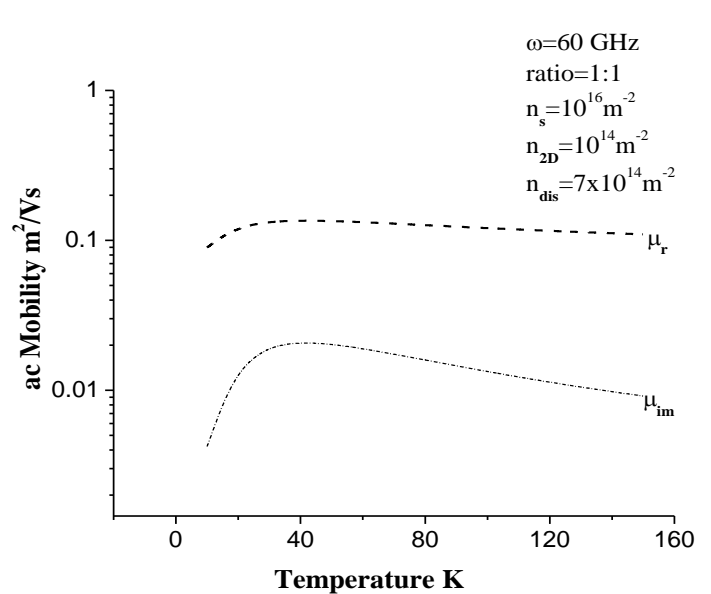

Fig. 6

Fig. 5: Variation of dc mobility versus temperature. Here $\mu_{\text {hetero }}$ is the mobility of $2 \mathrm{D}$ EG in the heterojunction taking it as a single layer, $\mu_{1: 1}$ and $\mu_{24: 1}$ are the combined two layer mobilities with the junction to the dislocation layer thickness ratio to be 1:1 and 24:1 respectively. Whereas $\mu_{\exp }$ is the experimental result of Gokden [10].

Fig. 6: Variation of two layer ac mobility versus temperature, taking the ratio of the junction to the dislocation layer thickness to be $1: 1$ at constant frequency $60 \mathrm{GHz}$. The curves indicated by $\mu_{\mathrm{r}}$ and $\mu_{\mathrm{im}}$ show the combined real and imaginary parts of the ac mobility respectively.

\section{Conclusions}

The electronic transport parameters such as dc and ac mobilities on heterojunction of $\mathrm{AlGaN} / \mathrm{GaN}$, grown on sapphire is calculated on the basis of two-layer model, formulated by Look is calculated. 
The various scattering mechanisms such as acoustic phonon (ac), piezoelectric (pz), surface roughness (sr) and ionize impurity (ii) scatterings are taken into consideration in the heterojunction layer whereas only the dislocation (dis) scattering is taken into account in the interfacial layer between GaN and the sapphire.

The two layer combined mobility curve for the thickness of the heterojunction layer equal to the interfacial layer in the low temperature range is found comparable to the experimental result[10]. At high temperature range, on the inclusion of the polar optical scattering (pop) becomes dominant but in the present work, it is not taken into account. However, the presence of the interfacial or dislocation layer can't be ignored.

On the basis of the results obtained for the thickness ratio 1:1, it can be concluded that the two layer model as suggested by D.C Look supports the experimental results.

\section{Acknowledgement}

One of the authors S. Shrestha is grateful to Indian National Science Academy (INSA), New Delhi, India for providing the INSA-JRD-Tata Fellowship to carry out the above research work.

\section{References}

[1] R.L. Anderson, Germanium-Gallium Arsenide Heterojunctions, IBM.J. Res. Dev. 4 (1960) 283-287. doi.org/10.1147/rd.43.0283

[2] F. Stern, W.E. Howard, Properties of Semiconductor Surface Inversion Layers in the Electric Quantum Limit, Phys. Rev. 163 (1987) 816. doi.org/10.1103/PhysRev.163.816.

[3] J. D. Zook, Piezoelectric Scattering in Semiconductors, Phy. Rev. 136 (1964) A869. doi.org/10.1103/PhysRev.136.A869.

[4] D.C. Look, J.R. Sizelove, Dislocation Scattering in GaN, Phys. Rev. Lett. 82 (1999) 1237. doi.org/10.1103/PhysRevLett.82.1237.

[5] D.C. Look, R.J. Molnar, Degenerate layer at GaN/sapphire interface: Influence on Hall-effect, Appl. Phys. Lett. 70 (1997) 3377. doi.org/10.1063/1.119176.

[6] C.K. Sarkar, P. Banerji, Estimation of the Alloy Scattering Potential in . - Wiley Online Library, Phys. Stat. Sol. (b), 156 (1989) K145. doi.org/10.1002/pssb.2221560248.

[7] D.C. Look, Electrical Characterization of GaAs Materials and Devices, Johan and Sons Ltd., NY, 1989.

[8] B.R. Nag, Electron Transport in Compound Semiconductors Vol. II, edited by H.J. Queisser, Springer Berlin, 1980.

[9] D. Jena, A.C. Gossard, U.K. Mishra, Dislocation scattering in a two-dimensional electron gas. Department of Electrical and Computer Engineering. University of California. Santa Barbara, California, 93106, 2000.

[10] S. Gokden, Dislocation scattering effect on two-dimensional electron gas transport in GaN/AlGaN modulation-doped heterostructures. Elsevier. Physica E 23 (2003) $19-25$. 